\title{
Dynamic TXOP HCCA reclaiming scheduler with transmission time estimation for IEEE 802.11 e real-time networks
}

\author{
Gabriele Cecchetti \\ g.cecchetti@sssup.it
}

\author{
Anna Lina Ruscelli \\ a.ruscelli@sssup.it
}

\author{
Giuseppe Lipari \\ giuseppe.lipari@Isv.ens- \\ cachan.fr
}

\author{
Antonia Mastropaolo \\ antonella.mastropaolo@gmail.com \\ ReTiS Lab. - TeCIP \\ Scuola Superiore S. Anna \\ Pisa, Italy
}

\begin{abstract}
IEEE 802.11e HCCA reference scheduler guarantees Quality of Service only for Constant Bit Rate traffic streams, whereas its assignment of scheduling parameters (transmission time TXOP and polling period) is too rigid to serve Variable Bit Rate (VBR) traffic.

This paper presents a new scheduling algorithm, Dynamic TXOP $H C C A$ (DTH). Its scheduling scheme, integrated with the centralized scheduler, uses both a statistical estimation of needed transmission duration and a bandwidth reclaiming mechanism with the aim of improving the resource management and providing an instantaneous dynamic Transmission Opportunity (TXOP), tailored to multimedia applications with variable bit rate. Performance evaluation through simulation, confirmed by the scheduling analysis, shows that DTH is suitable to reduce the transmission queues length. This positively impacts on the delay and on packets drop rate experienced by VBR traffic streams.
\end{abstract}

\section{Categories and Subject Descriptors}

C.2.1 [Computer-Communication Networks]: Network Architecture and Design-Wireless Communication

\section{General Terms}

Algorithms, Performance

\section{Keywords}

Quality of Service, real-time scheduling algorithms, wireless LAN

Permission to make digital or hard copies of all or part of this work for personal or classroom use is granted without fee provided that copies are not made or distributed for profit or commercial advantage and that copies bear this notice and the full citation on the first page. To copy otherwise, to republish, to post on servers or to redistribute to lists, requires prior specific permission and/or a fee.

MSWiM'12, October 21-25, 2012, Paphos, Cyprus.

Copyright 2012 ACM 978-1-4503-1628-6/12/10 ...\$15.00.

\section{INTRODUCTION}

The wide spreading of multimedia applications over Wireless Local Area Networks (WLAN) is a challenge for the resource management in order to provide the required Quality of Service (QoS). Multimedia streaming, Voice Over IP (VoIP), High Definition TV (HDTV), mobile applications etc. require a service differentiation based on tailored specification of QoS parameters like end-to-end delay, jitter, packet loss etc.

IEEE 802.11e amendment [19] to IEEE 802.11b [18] adds $H y$ brid Coordination Channel Access (HCCA) MAC protocol, a parameterized polling mechanism. Each Station with QoS (QSTA) negotiates the service that better meets the requirements of its Traffic Streams (TSs) with QoS Access Point (QAP).

The service differentiation of IEEE 802.11e reference scheduler, whose guidelines are drawn by the standard, is performing for Constant Bit Rate (CBR) TSs, but not for Variable Bit Rate (VBR) TSs, as shown by numerous studies $[10,16,27,33]$. Indeed, the reference scheduler suggests computing the main protocol parameters, during the admission control: a fixed Transmission Opportunity $T X O P_{A C, i}$ for each $\mathrm{QSTA}_{i}$ and a same Service Interval SI, (the polling period), for all stations. Hence, it provides a sufficient but not optimal service, set around mean values streams parameters, but this is not satisfying for VBR traffic, where bit data rate can vary with respect to its mean value, and where traffic periods can be different.

Traffic variations require a flexible resource management, in order to better follow the traffic profile. For instance, in the case of highly variable bit rate, when data rate falls below its average value, the assigned $T X O P_{A C}$ is not exhausted. Instead, when data rate goes up and overcomes its mean value, the accorded transmission time is not sufficient to dispatch the incoming streams and to absorb bursts of traffic. Consequently, transmission queues length increases, jeopardizing the assured QoS and real-time network performance, expressed in terms of end-to-end delay, is worse. Also the packets drop rate, due to expiration of packets validity time, rises. A very simple solution is allocating more resources than needed in normal conditions. However, overprovisioning reduces the number of QSTAs admitted to transmit, and wastes resources when there is no high data rate. Also dynamically modify, at each polling, the TXOP computation can yet jeopardizes the admission control feasibility test. Instead, a method suitable for providing a 
dynamic TXOP, without changes in the accorded resources threshold, is a bandwidth reclaiming scheme $[6,9,24,28,30]$.

In this paper, a new scheduling algorithm Dynamic TXOP HCCA (DTH) is presented. DTH integrates a bandwidth reclaiming mechanism into a centralized HCCA scheduler, improving the transmission capacity management and providing further resources to VBR TSs, when needed. It is able to recover the unused portion of transmission time and assign that to the next polled station, which can need a longer transmission interval, and it prevents losing the spare time. Hence, DTH computes an instantaneous dynamic TXOP, tailored to the considered traffic type. This computation is further refined introducing an estimation of the previously used transmission time of each QSTA, with the aim of adapting the currently assigned TXOP to the effective resource needs. Moreover, since DTH takes action only at the polling time for computing the current $T X O P$, it does not modify the centralized scheduling policy. Finally, since it simply recovers unused time, it does not overcome the maximum threshold of the accorded resources, set during the admission control.

Analytical study and performance evaluation through simulation will show that DTH is able to reduce the transmission queues length and delay, improving real-time performance and following traffic variations.

This paper is organized as follows: in Section 2 a brief summary of most meaningful HCCA schedulers with QoS is listed, in Section 3 the DTH scheduling algorithm is illustrated, whereas in Section 5 performance evaluation through simulation is shown. Finally Section 6 concludes the work.

\section{RELATED WORKS}

The IEEE 802.11e HCCA reference scheduler [19] suggests how to compute the main protocol parameters: polling period Service Interval (SI), and transmission duration Transmission Opportunity (TXOP). They are calculated as fixed values during the admission control phase and are recomputed only if a new Traffic Stream (TS) is admitted to transmit.

Numerous studies about the reference scheduler, [10,16, 27,33], shown that it is suitable to serve only CBR traffic, whereas it is unable to efficiently manage VBR TSs, since all different TSs are polled with the same period and are served with the same computation time. Hence, many alternative scheduling algorithms have been proposed to improve the HCCA QoS provisioning [12, 14,26, 33 , but the support of the real-time requirements has been investigated only by few studies [4]. In the following, at the best of our knowledge, we summarize some significant HCCA real-time schedulers.

Fair HCF (FHCF) [1] computes variable TXOPs by means of a mathematical model that distinguishes between packet queuing delay, influenced by the variations in packet size and data rate, and waiting time delay until the polling time.

Feedback Based Dynamic Scheduler (FBDS) [3] uses a closed loop feedback control to limit the maximum delay. The queue length estimation corrected by the queue length information sent by each QSTA, is used to dynamically calculate TXOPs; $S I$ is fixed.

Most of the works based on IEEE 802.11e HCCA-EDCA Mixed Mode (HEMM) are focused on the optimization of the HCCAEDCA duration ratio to efficiently manage multimedia traffic over both these functions $[22,23,31]$.

Instead Overboost [29] uses EDCA resource to improve the HCCA traffic scheduling. This local node scheduler moves the traffic exceeding the assigned HCCA TXOP from the HCCA queue to the higher priority Access Category EDCA queue, collaborating with the centralized HCCA scheduler that continues to manage admission control, scheduling parameters, and polling list.

Scheduling Estimated Transmission Time - Earliest Due Date (SETT-EDD) [15] uses a token bucket scheme to vary TXOPs according to the station requirements. Each $S I$ is computed taking into account its traffic profile, while Earliest Deadline First (EDF) [25] determines the polling order.

Fan [11] uses a timer-based scheduler to compute the transmissions deadlines as the smallest between the downlink and uplink ones, and EDF schedules traffic streams.

Real-Time HCCA (RTH) scheduler [8] manages TXOP as critical section by using Stack Resource Policy (SRP) algorithm [2]. An EDF-based algorithm schedules TS transmissions.

Adaptive Resource Reservation Over WLANs (ARROW) [32] dynamically computes each $T X O P$ by taking into account the actual different buffered TSs data at the beginning of the polling. Moreover, Maximum SI is bounded in order to ensure no deadline miss and delay requirements. Finally Earliest Due Date (EDD) [21] manages the QSTAs polling list.

Application-Aware Adaptive HCCA Scheduler [20] distinguishes uplink and downlink schedulers, while EDF [25] defines the polling order. The uplink scheduler assigns each QSTA a minimum and a maximum $S I$, adapted to application and network conditions and to the buffered traffic.

Wireless Capacity-Based Scheduler (WCBS) [5] dynamically updates QSTA polling list using EDF algorithm and uses static and dynamic parameters to adapt the transmissions scheduling to the TSs characteristics.

All these mentioned schedulers try their best to compute variable TXOP and/or SI in order to improve the VBR traffic management, but they do not recover assigned but not used resources, that could be present in the case of highly variable TSs.

The bandwidth reclaiming mechanism proposed by Larcheri [24] uses two different max-min fairness algorithms to reduce the delay produced by HCCA in the case of VBR traffic by recovering spare resources through a proportional controller.

Its non linearities are overcame by Palopoli [28] with an optimal controller, based on Model Predictive Control [13].

Moreover Lo Cigno applies [9] both cited proportional controller as optimal controller algorithms to multi-class traffic with different priorities and to heterogeneous traffic.

Unused Time Shifting Scheduler [30] is based on a greedy reclaiming scheme that recovers the unspent time from previous polled stations and assigns that to the next polled one in order to provide further resources to serve bursty traffic.

\section{DYNAMIC TXOP HCCA SCHEDULER}

Traffic dynamicity of VBR applications suggests an adaptive computation of the TXOP protocol parameter.

Modifying $T X O P$ by finding additional resources, without upgrading the assigned resources threshold but recovering the unused ones, can be obtained knowing the effectively used transmission time at each polling and foreseeing the needed one, and evaluating the presence of resources that can be recovered.

As far as transmission time assignment is concerned, sending duration is dependent of the traffic profile, in particular of the bit data rate. Thus, if the traffic profile is unknown, as in the case of TSs transmission, analyzing the trend of used transmission time is functional to compute the suitable $T X O P^{\prime}$. Moreover IEEE 802.11e HCCA helps to find further resources, without implying a global assignment recomputation. Indeed, QAP takes control of the medium when a station has exhausted its transmission time, or when it has no more data to send and its TXOP is not finished. In 


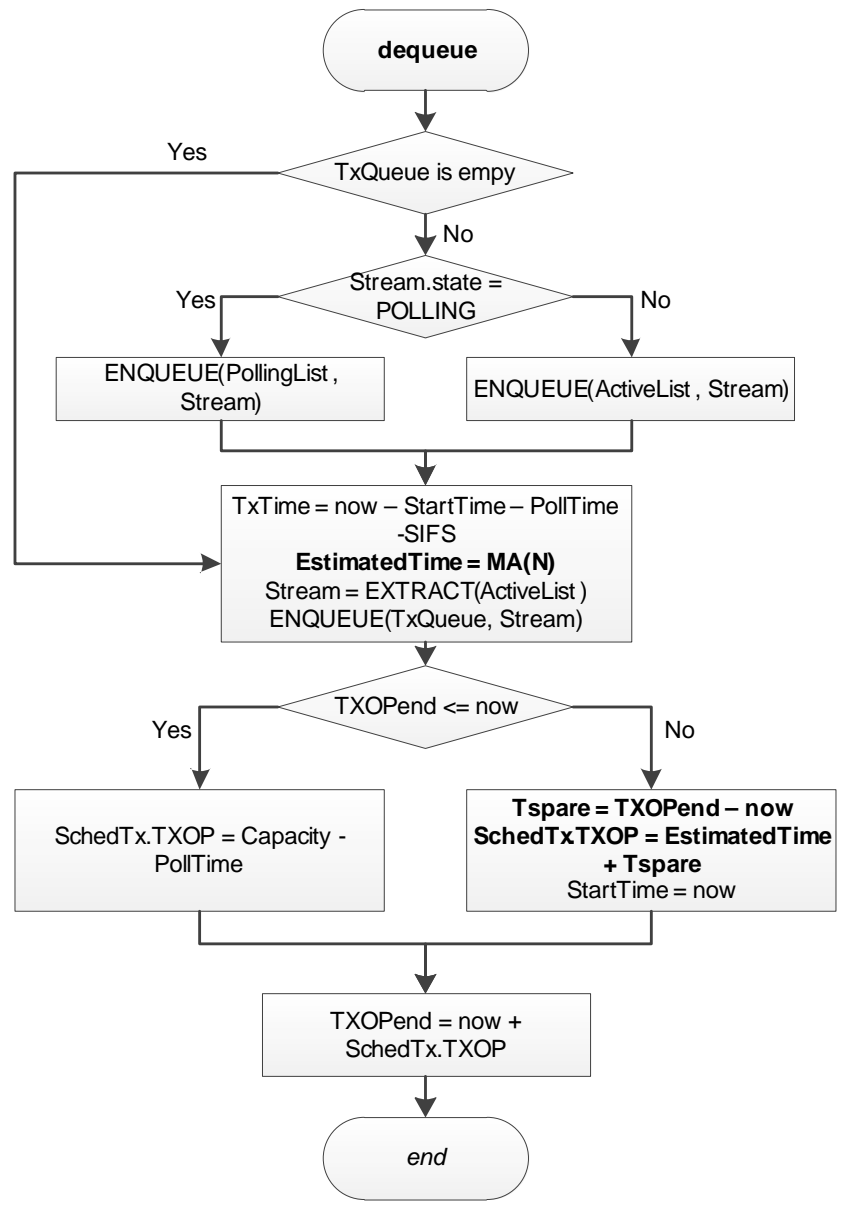

Figure 1: DTH dequeue flow diagram

this last situation the remaining portion of transmission opportunity is no more used and can be reclaimed.

These are the basic ideas behind Dynamic TXOP HCCA algorithm. DTH aims at integrating the centralized scheduler with an additional mechanism, exclusively focused on the computation of an instantaneous TXOP $P_{i}^{\prime}$ appropriate to the scheduled $T S_{i}$. DTH does not deal with general protocol parameters computation, polling list sorting, polling of stations, access medium management. These tasks are still performed by the QAP scheduling algorithm, as it will be demonstrated in Section 4.

In particular, DTH is composed by two different mechanisms, shown in bold style in Fig. 1 that summarizes the function dequeue used to extract a TS from the polling queue and assign the polling time and the transmission time. The first, which deals with the estimation of effectively used transmission time, aims at refining the current $T X O P$ allocation. It tries to foresee the transmission time that will be effectively used during the next polling, studying the previously used transmission intervals. The second mechanism is focused on finding the spare transmission time.

\subsection{Statistical estimation of needed transmis- sion time}

The estimation of the transmission time expected to be effectively used during the next polling is inspired by the concept of time series. This is used in statistics, signal processing, econometrics and mathematical finance, to study a stochastic phenomenon by monitoring its temporal behavior. A time series is composed by a set of temporally ordered random variables, with the aim at describing its temporal variations. This theoretical tool is perfectly suitable to describe the trend of transmission duration and, consequently, to foresee the length of the next sending interval. Thus, the effectively used transmission time $\Delta$ of QSTA $_{j}$ is described by the time series:

$$
\Delta=\left\{t_{\text {used }}, t, t \in T\right\},
$$

where the time $t$ is the state variable, $t_{\text {used }}$ is the observation of $\Delta$ at time $t$, and $T$ is the discrete index set, that is referred to the time periods, i.e., the sampling periods. In our case they are coincident with traffic periods, since we assume as sample each transmission of the considered QSTA during subsequent Controlled Access Phases (CAPs). In time series forecasting Moving Average (MA) is suitable to obtain an evaluation of the trend component of an investigated phenomenon from the more recent samples $y_{i}$. MA of order $m_{1}+m_{2}$ at time $t$ is expressed as follows:

$$
M A(t)=\sum_{i=-m_{1}}^{m_{2}} \theta_{i} \cdot y_{t+i}
$$

where $m_{1}$ is the number of periods before $t, m_{2}$ after $t$, and $\theta_{i}$ is the weight of the element $i$. In the case of DTH all samples have the same importance, thus $\theta_{i}=\frac{1}{m_{1}+m_{2}}, \forall i$. Moreover, since all samples of a $\mathrm{QSTA}_{j}$ have the same period, $S I_{j}$, the Simple Moving Average (SMA) is used. Its computation is coincident with that of the arithmetic average, except that it uses a mobile window to collect the samples. Thus, $t_{\text {est }}(t)$, the estimated transmission time of a station $j$ at time $t$, before the $(N+1)^{t h}$ polling, is computed through the SMA of the effectively used transmission time during the previous $N$ polling:

$$
t_{\text {est }}(t)=\frac{\sum_{i=1}^{N} t_{\text {used }_{j, i}}}{N},
$$

where $t_{u s e d_{j, i}}$ is the transmission time effectively used during the $i^{t h}$ polling time by $\mathrm{QSTA}_{j}$, see Figure 2. In this computation the index set $T$, and, then, the length of observations interval, the sampling window, are chosen dependently from the traffic type and period, in order to have a sufficient number of samples. For instance, if we assume that $T$ is composed by 250 elements, in the case of a video streaming TS with a period of $40 \mathrm{~s}$, the sampling window is long $10 \mathrm{~s}$. Then, shifting the window along the time axis is possible to have an updated estimation of the transmission time used during the last 250 polling.

This efficient approach can be improved through the integration of a mechanism to recover the unspent transmission time. Moreover this method helps to diagnose the inversion in the data rate trend.

\subsection{Resources recovery computation}

The time remaining from the previous transmission can provide the additional resources needed when data rate exceeds its average value, without overprovisioning.

The unused time to recover, $T_{\text {spare }}$, is computed with the same mechanism of UTSS, as follows:

$$
T_{\text {spare }}=t_{\text {end }}-t_{\text {stop }}
$$

where $t_{\text {end }}=t_{p}+$ TXOP is the ending transmission time when $T X O P$ is completely exhausted ( $t_{p}$ is the polling time), and $t_{s t o p}$ is the actual finishing transmission time. 


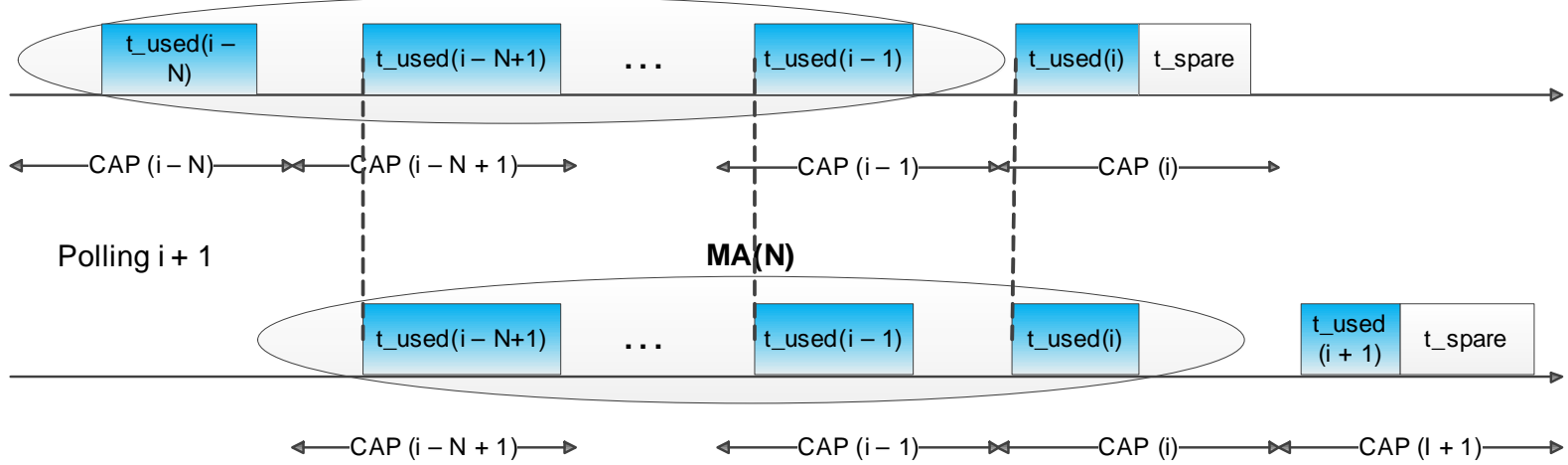

Figure 2: Transmission time estimation mechanism.

However the statistical evaluation of the current transmission time makes the DTH TXOP computation completely different. Indeed, in UTSS, no estimation mechanism was adopted and $T_{\text {spare }}$ was simply shifted to the next polled station, without verifying if needed or not. This means that it was likely to assign the recovered time to station that did not use that and, consequently, the same $T_{\text {spare }}$ had to be taken twice. Instead DTH tries to avoid unnecessary assignments. It makes the dynamic TXOP computation more precise with respect to the traffic trend.

\subsection{Transmission Opportunity computation}

Finally, $T X O P_{i}^{\prime}$ for the next polling of $Q S T A_{i}$ is computed as follows:

$$
T X O P_{i}^{\prime}= \begin{cases}T X O P_{A C(i)} & \text { if } T_{\text {spare }} \equiv 0 \\ t_{\text {est }(i)}+T_{\text {spare }} & \text { if } T_{\text {spare }}>0\end{cases}
$$

\section{DTH SCHEDULER ANALYSIS}

In this section an analytical study of DTH is presented. It aims at investigating its properties and the impact of its integration with a centralized scheduling algorithm on the real-time properties of the global scheduler. Hence, first of all, it is shown that the assigned $T X O P^{\prime}$ is tailored to follow traffic variability. Then DTH statistical features are analyzed. As far as real-time properties are concerned the effect of the bandwidth reclaiming scheme and of the transmission time estimation on the admission control feasibility test and on the respect of deadlines constraints is studied.

PROPOSITION 1. Setting TXOP ${ }_{i}^{\prime}=T X O P_{A C, i}$ when $T_{\text {spare }}=0$ is suitable to avoid saturation effect when data rate begins growing and only $t_{\text {est }}$ is considered and, then, it is suitable to efficiently manage variations of VBR streams data rate.

PROOF. In order to highlight the scheduler behavior without the resource integration mechanism, assume $T_{\text {spare }}=0$. Fig. 3 illustrates the centralized scheduler + DTH behavior, first, when data rate $R$ decreases and, then, when increases.

At $i^{\text {th }}$ polling the assigned $T X O P_{i}^{\prime}$ is:

$$
\operatorname{TXOP}_{i}^{\prime}=t_{\text {est }(i)}=\frac{\sum_{j=i-N}^{i-1} t_{u s e d(j)}}{N} .
$$

At $(i+1)^{t h}$ polling $T X O P^{\prime}$ is:

$$
T X O P_{i+1}^{\prime}=t_{e s t(i+1)}=\frac{\sum_{j=i-N+1}^{i} t_{u s e d(j)}}{N} .
$$

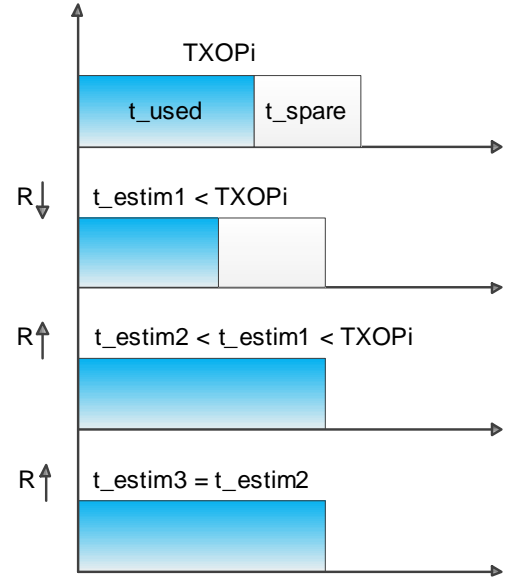

Figure 3: Example of saturation.

If $R$ is decreasing, $T X O P_{i+1}^{\prime}<T X O P_{i}^{\prime}$ since MA is based on samples from $i-N+1$ to $i+1$, included $t_{\text {used }(i)}<T X O P_{i}^{\prime}$, thus the average decreases. The same behavior happens for $(i+2)^{t h}$ polling: correctly the scheduler reduces the assigned transmission time in order to follow $R$, which is decreasing. But if at polling $(i+3)^{t h} R$ changes its trend and begins increasing, $t_{\text {used }(i+3)} \equiv T X O P_{i+3}^{\prime}$. If $R$ continues to grow and we continue to assign $T X O P_{i}^{\prime} \equiv t_{\text {used }(i)}$, i.e. we continue to assign $T X O P^{\prime}$ considering only the estimation of previously used transmission time, at next polling

$$
T X O P_{i+4}^{\prime}=t_{e s t(i+4)}=\frac{\sum_{j=i-N+3}^{i+3} t_{u s e d}(j)}{N}>T X O P_{i+3}^{\prime},
$$

and $t_{\text {used },(i+4)} \equiv T X O P_{i+4}^{\prime}$ since now all assigned resources are necessary. If at polling $(i+5)^{t h}$, assuming $R$ is yet increasing,

$$
T X O P_{i+5}^{\prime}=t_{e s t(i+5)}=\frac{\sum_{j=i-N+4}^{i+4} t_{u s e d}(j)}{N} \equiv T X O P_{i+4}^{\prime} .
$$

from this point onwards, as shown, if $R$ is increasing, the mechanism is saturated since, at each polling, QSTA uses the whole accorded transmission time but, if $T_{\text {spare }}=0$, there is no chance to upgrade $T X O P^{\prime}$. Hence, the choice of assigning $T X O P_{A C, i}$ when $T_{\text {spare }}=0$ is a reasonable tradeoff in order to follow traffic variability and, in particular, changes of $R$ trend. Indeed, even if this solution is superabundant when $R$ decreases, when data rate in- 
creases and random recovered resources are not present, it provides the maximum allowed capacity. This is a prudential method since the algorithm is primarily focused on preserving real-time performances as well as saving resources. Furthermore it is possible to highlight that DTH shows a certain inertia, which enables to ignore traffic spikes and to follow the general traffic trend.

THEOREM 1. The admission control feasibility test is respected when the DTH mechanism is integrated with the centralized scheduler.

Proof. Assume to consider the admission control feasibility test suggested by the HCCA reference scheduler. It is expressed as follows:

$$
\sum_{i=1}^{M} T X O P_{i}=T_{C A P}=T_{H}-T_{C P}
$$

where $T_{C A P}$ is the CAP length, $T_{H}$ is the duration of the hyperperiod and $T_{C P}$ is that of Contention Period, and $M$ is the number of stations composing the Basic Service Set related to the considered QAP and admitted to transmit. Without loss of generality, assuming to allow $T_{\text {spare }}$ propagation between the last polled station during a CAP and the first one at the subsequent CAP, when DTH is used, at $j^{\text {th }}$ CAP the admission control expression becomes:

$$
\sum_{i=1}^{M} T X O P_{i}=\sum_{i=1}^{M}\left\{t_{\text {est }(i)}+T_{\text {spare }(i)}-T_{\text {spare }(i+1)}\right\}=\sum_{i=1}^{M} t_{\text {est }(i)}
$$

where $T_{\text {spare }, i}$ is the recovered time assigned to $\mathrm{QSTA}_{i}$, and $T_{\text {spare, }(i+1)}$ is the time unused by station $i^{t h}$ and shifted to station $(i+1)^{t h}$. The expression holds since $T_{\text {spare }}$ is simply propagated along the QSTAs chain. Moreover, since $t_{e s t, i}$ is function of used transmission time during the previous $N$ CAPs,

$$
\begin{aligned}
\sum_{i=1}^{M} t_{\text {est }(i)=} & \sum_{i=1}^{M} M A_{i}(t) \\
= & \sum_{i=1}^{M}\left\{\sum_{l=j-N}^{j-1} t_{\text {used }(i, l)}\right\} \\
= & \sum_{l=j-N}^{j-1} \frac{t_{\text {est }(1, l)}+T_{\text {spare }(1, i)}-T_{\text {spare }(2, i)}}{N}+ \\
& \sum_{l=j-N}^{j-1} \frac{t_{\text {est }(2, l)}+T_{\text {spare }(2, i)}-T_{\text {spare }(3, i)}}{N}+\ldots+ \\
& \sum_{l=j-N}^{j-1} \frac{t_{\text {est }(M, l)}+T_{\text {spare }(M, i)}-T_{\text {spare }(1, i)}}{N} \\
\leq & T_{C A P}=T_{H}-T_{C P}
\end{aligned}
$$

since during each one of the $N$ polling considered by MA $T_{\text {spare }}$ is propagated between the polled QSTAs.

THEOREM 2. The global scheduler, composed by the centralized algorithm and DTH, continues meeting deadlines.

PROOF. Independently from the type of $T_{\text {spare }}$ propagation, if or not enabled between consecutive CAPs, DTH does not modify the centralized scheduling policy. In the case of $T_{\text {spare }}>0$ it simply shifts that to the next polled station. This means that this station receives an upgrade of resources and is polled in advance of the same $T_{\text {spare }}>0$, with respect to the polling time without DTH. Since the portion of added capacity is added polling in advance of the same time interval the station, then it is possible to deduct that deadlines are respected.
In order to investigate the impact of DTH on delay and packets drop rate, the behavior of queues length is analyzed.

THEOREM 3. The introduction of DTH is suitable to reduce transmission queues length.

Proof. In order to highlight the effect of an increasing data rate $R$ on the transmission queues length, the traffic arriving from the previous transmission ending the ratio $\eta$ between the incoming traffic during two subsequent polling is considered. Without loss of generality we assume no residual traffic from previous polling, thus queue length is due to the incoming streams. If at polling $i^{\text {th }}$ $R \equiv R_{\text {mean }}$, and at polling $(i+1)^{\text {th }} R>R_{\text {mean }}$, in worst condition, at polling time $t_{i} \eta_{i}$ is equal to:

$$
\eta_{i}=\frac{\left(t_{i}-t_{\text {end }(i-1)}\right)\left(R_{\text {max }}-R_{\text {mean }}\right)}{\left(t_{i}-t_{\text {end }(i-1)}\right) R_{\text {mean }}}>1,
$$

where the traffic arriving from the previous transmission ending time $t_{\text {end }(i-1)}$ and the current time $t_{\text {now }}$ are considered. $R_{\max }$ is the max value of data rate reached during the considered interval. Thus, transmission queue length increases. However, when $T_{\text {spare }}>0$, part of this further traffic can be dispatched using this added capacity. This introduces a reduction of transmission queue length with respect to no use of DTH. Indeed, since the delay experience by packets to send is due both to transmission duration that to waiting time into the transmission queues, DTH positively affects the delay experienced by TSs, as it will be shown in Section 5 .

\section{EXPERIMENTAL RESULTS}

This section presents the results of DTH performance evaluation obtained through simulations.

We first describe the simulation settings and the used traffic model. Then we discuss the analysis results about admission control, polling interval, and experienced null rate in order to investigate the effect of DTH on the centralized scheduling algorithm. Then transmission queues length and mean access delay are analyzed. The obtained results are compared with reference and WCBS schedulers. Indeed, being WCBS an EDF-based scheduler, it does not show good delay performances [4], thus it is a candidate suitable to highlight the DTH effects on real-time performances.

\subsection{Simulation settings, network scenario and traffic model}

The simulations has been carried out using Network Simulator 2 (ns-2) [17], with the HCCA extension described in [7].

The Physical layer is specified in IEEE 802.11g standard, which uses OFDM (Orthogonal Frequency-Division Multiplexing) as mandatory modulation scheme; its parameters are listed in Table 1.

MAC level fragmentation, multirate support, RTS/CTS protection mechanism are disabled and we assume that all nodes can directly communicate with each other, without the hidden node problem.

\begin{tabular}{lrlr}
\hline Parameters & Value & Parameters & Value \\
\hline SIFS $(\mu s)$ & 10 & PLCP header $(b)$ & 24 \\
DIFS $(\mu s)$ & 28 & Preamble $(b)$ & 72 \\
PIFS $(\mu s)$ & 19 & Data Rate $($ Mbit $/ s)$ & 54 \\
Slot Time $(\mu s)$ & 9 & Basic Rate $($ Mbit $/ s)$ & 1 \\
\hline
\end{tabular}

Table 1: MAC/PHY simulation parameters.

The presented results have been obtained simulating independent replications of $700 \mathrm{~s}$ with a warm-up time of $100 \mathrm{~s}$ until the 
95\% confidence interval is reached for each measure. Confidence intervals are not drawn whenever negligible.

The chosen scenario is composed by one QAP and five QSTAs. Each station transmits one uplink TS, which is received by QAP. QSTA named VP sends VoIP traffic encoded with G.729A codec, whose parameters are shown in Table 2.

\begin{tabular}{lrlr}
\hline Parameter & Value & Parameter & Value \\
\hline Frame size $(B)$ & 10 & Payload size $(B)$ & 20 \\
Frame per packet & 2 & IP/UDP/RTP & \\
Period $(s)$ & 0.02 & Header size $(B)$ & 40 \\
Data rate $(k b / s)$ & 24 & SDU size $(B)$ & 60 \\
\hline
\end{tabular}

Table 2: G.729A VoIP traffic stream parameters.

QSTA named $V C$ transmits a pre-encoded video conference session. The remaining QSTAs, from VS1 to VS3, stream pre-encoded high quality MPEG4 videos. The traffic specifications of these four stations are listed in Table 3.

\begin{tabular}{lrrrr}
\hline Parameter & VC & VS1 & VS2 & VS3 \\
\hline Mean frame size (B) & 3800 & 3800 & 2900 & 2900 \\
Maximum frame size (B) & 11386 & 11386 & 22239 & 15251 \\
Period (s) & 0.040 & 0.040 & 0.040 & 0.040 \\
Mean data rate (kb/s) & 770 & 770 & 580 & 580 \\
Maximum data rate $(\mathrm{kb} / \mathrm{s})$ & 3300 & 3300 & 4400 & 3100 \\
\hline
\end{tabular}

Table 3: Video streaming and video conference parameters.

\subsection{Performance evaluation}

The efficiency of HCCA schedulers can be evaluated looking at the number of the admitted TSs, null rate and polling interval.

Since DTH uses the same admission control test of WCBS thus the number of admitted TSs is the same, and it is higher than the one produced by the reference scheduler, due to improved efficiency in resource management.

Null rate is the sending rate of $\mathrm{CF}-\mathrm{Null}$ packets as answer to a polling when the station has no data to transmit; it is due to an uncorrect polling time and can increase the network overhead.

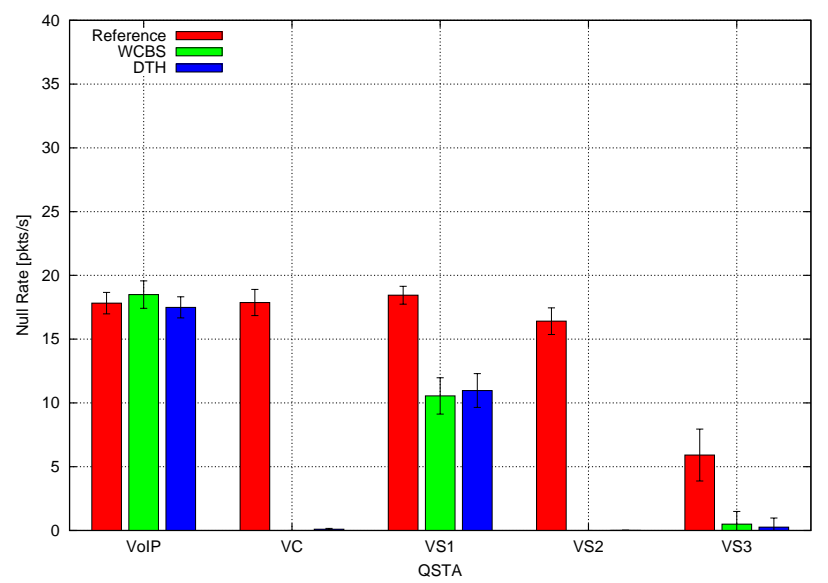

Figure 4: Null rate.

Fig. 4 shows as reference, WCBS, and DTH schedulers have similar results about null rate only for VoIP traffic. When VBR traffic is transmitted, DTH and WCBS obtain lower values since they assign $S I$ and $T X O P$ considering the QSTAs requirements.

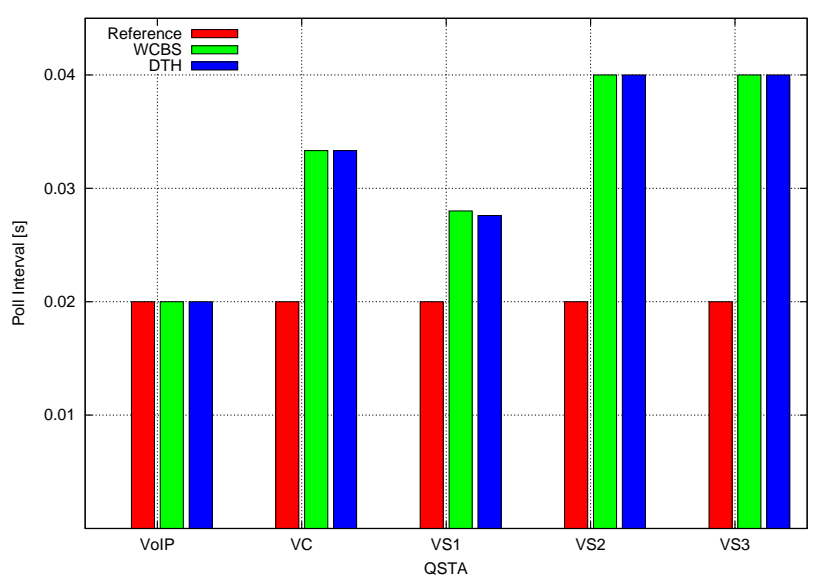

Figure 5: Polling interval.

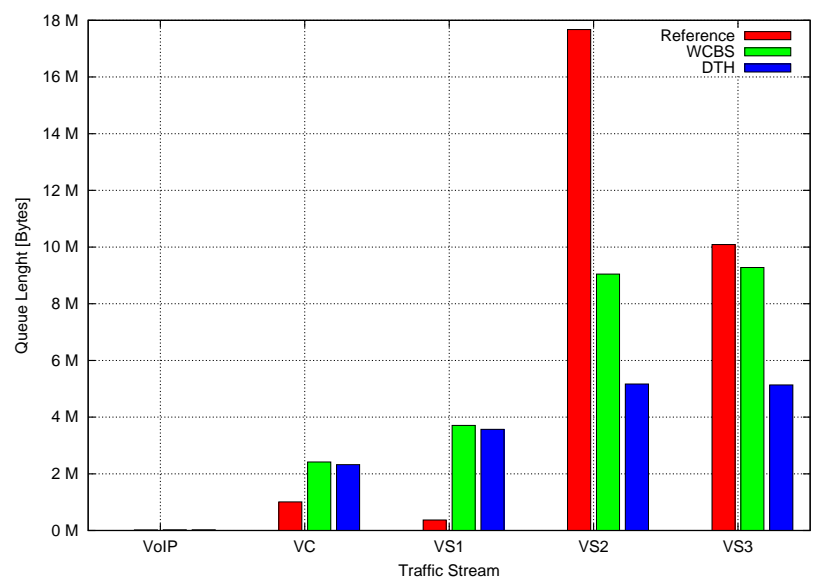

Figure 6: Traffic Stream queue length: 99th percentile.

In Fig. 5 the polling interval analysis is illustrated. As stated by the standard, reference scheduler polls all TSs with the same period. Instead DTH and WCBS are able to efficiently assign differentiated polling periods depending on traffic type. DTH provides values similar to those obtained by WCBS: the start time of each Contention Free Period is the same for both these algorithms, and, even if in DTH TXOP can vary over the time, the following shift of polling time for TSs belonging to the same CAP is almost negligible.

Fig. 6 shows the $99^{\text {th }}$ percentile of queue length for each TS. Since DTH assigns TXOP taking into account the average duration of recent transmissions and adding the further resources recovered through $T_{\text {spare }}$, it is able to transmit more packets, when need, taking advantage of this increased capacity, as deduced in Section 4. In this way the queues where packets wait to be transmitted are generally shorter. This improvement is particularly evident when a TS with an highly VBR, like VS2, is transmitted (in this case the DTH percentile is just $27 \%$ w.r.t reference and $55 \%$ of WBCS values). Fig. 7 focus the analysis on a single QSTA, VS3, in order to deeply study the effect of DTH on transmission queue length. In this case performance evaluation confirms that DTH is suitable to reduce queue length. In particular it limits HCCA queue at $5 \mathrm{MB}$ for about $90 \%$, and, in general, it has shorter queues than WCBS and reference schedulers for every percentage of the distribution. 


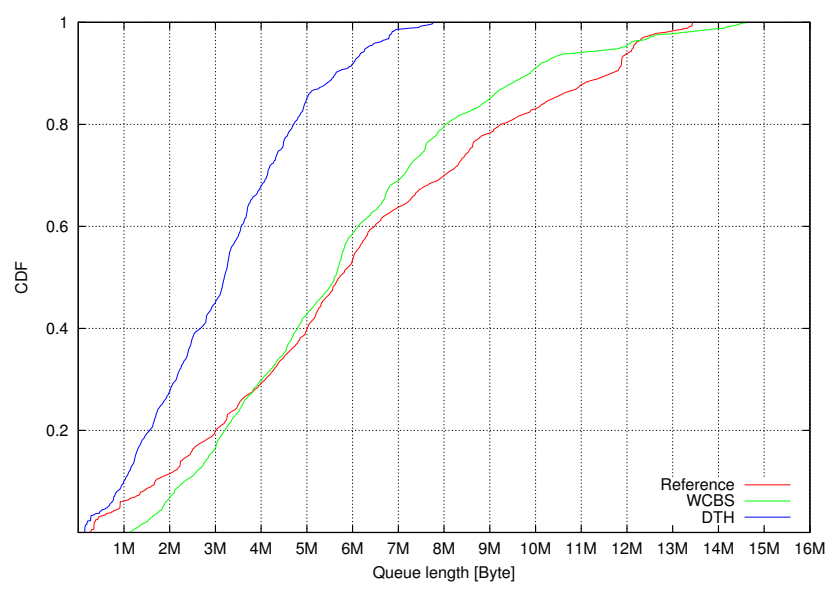

Figure 7: Queue length CDF for VS3 stream.

Throughput has been measured too but there are not significative differences among the considered schedulers.

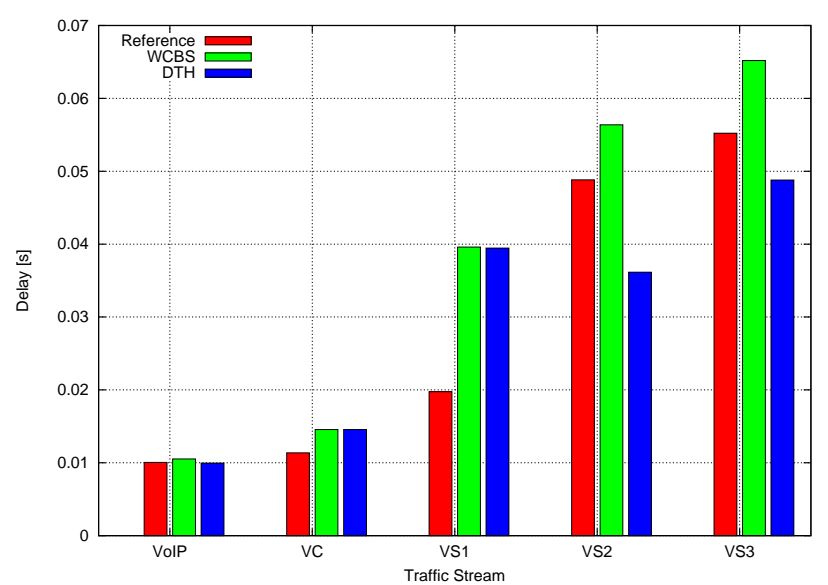

Figure 8: Mean Access Delay.

Mean access delay is illustrated in Fig. 8: here generally DTH performs as WCBS scheduler, except with high VBR TS, like VS3, where it is suitable to reduce access delay. This is due to the fact that it is able to adapt $T X O P$ to TS needs, providing more resources when needed.

Further investigating its behavior with VS3 by considering $\mathrm{Cu}$ mulative Distributed Function (CDF) of the access delay, Fig. 9 confirms that the DTH performs better respect to WCBS and reference.

Finally Fig. 10 shows the number of packets per second discarded at the QAP due to an experimented delay longer than the maximum allowed delay bound. DTH has the best performance compared to the other schedulers, and this matches the results obtained about queues length, and access delay.

\section{CONCLUSIONS}

In this paper a new HCCA scheduling algorithm, Dynamic TXOP $H C C A$ (DTH), has been presented. It is suitable to cooperate with the centralized scheduler in order to provide a more efficient mechanism to recover unused, but assigned, transmission time. Furthermore this recovery mechanism refines the computation of the

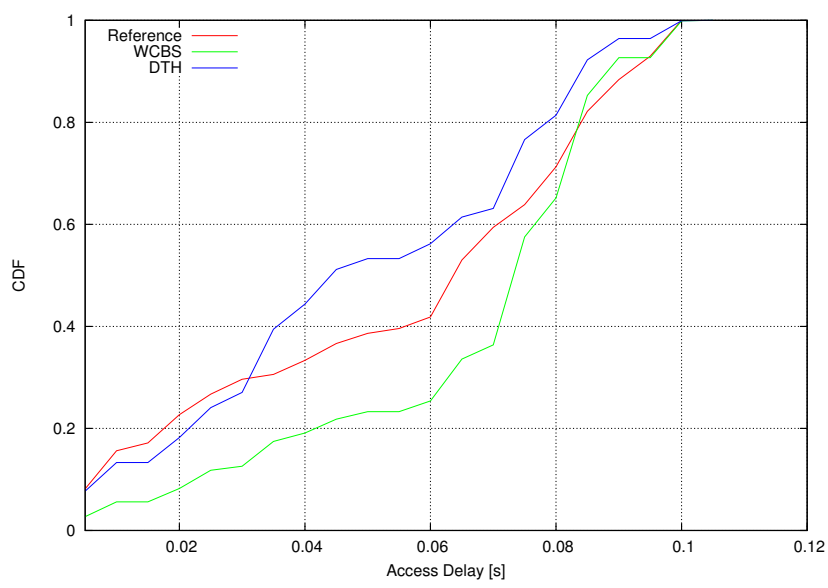

Figure 9: CDF of VS3 Access Delay.

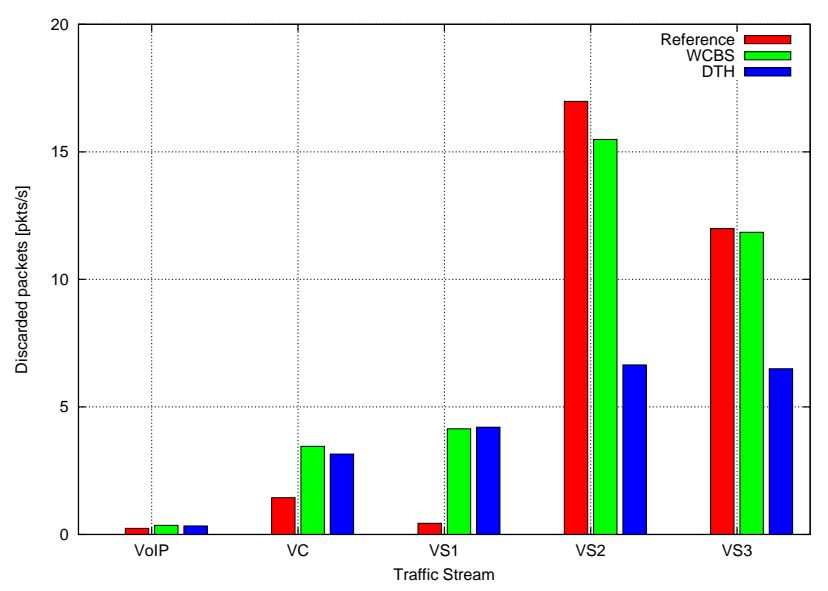

Figure 10: Discarded packets

current $T X O P$ considering the effective used transmission time of the current station during previous polling. It is computed using time series forecasting based on the Moving Average of the previous sending durations. This solution allows to assign a capacity tailored to the affective needs of the station.

Performance evaluation through simulation corroborated by the analytical study, confirms that DTH does not impact on the policy of the centralized scheduler but it is suitable to improve its performance in terms of transmission queues length, delay and number of dropped packets and absorb and follow data rate variations.

In the future works the use of the Moving Average to estimate the needed transmission time will be deeper investigated in order to find a setting of the mobile sampling windows more tailored to the different type of VBR traffic.

\section{Acknowledgements}

The research leading to these results has received funding from the European Community's Seventh Framework Programme under grant agreement n. 246556, "RBUCE-UP".

\section{REFERENCES}

[1] P. Ansel, Q. Ni, and T. Turletti. FHCF: A simple and efficient scheduling scheme for IEEE 802.11e wireless LAN. Mobile Networks and Applications, 11(3):391-403, June 2006. 
[2] T. P. Baker. Stack-based scheduling for real-time processes. Real-Time Syst., 3(1):67-99, 1991.

[3] G. Boggia, P. Camarda, L. G. Grieco, and S. Mascolo. Feedback-based control for providing real-time services with the IEEE 802.11e MAC. IEEE/ACM Trans. Net., 2(15):323-333, Apr. 2007.

[4] G. Cecchetti and A. L. Ruscelli. Real-Time support for HCCA function in IEEE 802.11e networks: a performance evaluation. Wiley Security and Communication Networks, 4(3):299-315, 2011. Special Issue on Security for QoS Assured Wireless Networks.

[5] G. Cecchetti, A. L. Ruscelli, and F. Checconi. W-CBS: a scheduling algorithm for supporting QoS in IEEE 802.11e. In Proceedings of IEEE/ACM QSHINE, pages 1-7, Vancouver, British Columbia, Canada, 2007.

[6] G. Cecchetti, A. L. Ruscelli, A. Mastropaolo, and G. Lipari. Providing variable TXOP for IEEE 802.11e HCCA real-time networks. In Proceedings of IEEE WCNC, Paris, France, Apr. 2012.

[7] C. Cicconetti, L. Lenzini, E. Mingozzi, and G. Stea. A software architecture for simulating IEEE 802.11e HCCA. In Proceedings of IPS MoMe, pages 97-104, Warsaw, Poland, Mar. 2005.

[8] C. Cicconetti, L. Lenzini, E. Mingozzi, and G. Stea. Design and performance analysis of the Real-Time HCCA scheduler for IEEE 802.11e WLANs. Computer Networks, 51(9):2311-2325, 2007

[9] R. L. Cigno, L. Palopoli, and A. Colombo. Analysis of different scheduling strategies in 802.11e networks with multi-class traffic. In Proceedings of IEEE LCN, Dublin, Ireland, Oct. 2007.

[10] J. Cowling and S. Selvakennedy. A detailed investigation of the IEEE $802.11 \mathrm{e}$ HCF reference scheduler for VBR traffic. In Proceedings of 13th ICC, pages 453-459, Chicago, US, Oct. 2004.

[11] Y. Fan and C. Huang. Real-Time traffic sheduling algorithm in WLAN. In Proceedings of 4GMF, San Diego, CA, 2005.

[12] H. Fattah and C. Leung. An overview of scheduling algorithms in wireless multimedia networks. IEEE Wireless, 9(5):76-83, Oct. 2002.

[13] C. Garcia, D. Prett, and M. Morari. Model predictive control: theory and practice: a survey. Automatica, 25(3):335-348, 1989.

[14] A. Grilo, M. Macedo, and M. Nunes. A service discipline for support of IP QoS in IEEE 802.11 networks. In Proceedings of PWC, Laapenranta, Finland, Aug. 2001.

[15] A. Grilo, M. Macedo, and M. Nunes. A scheduling algorithm for QoS support in IEEE 802.11e networks. IEEE Wireless Comm., 10(3):36-43, June 2003.

[16] A. Grilo and M. Nunes. Performance evaluation of IEEE 802.11e. In Proceedings of 13th IEEE PIMRC, volume 1, pages 511-517, Lisboa, Portugal, Sept. 2002.

[17] http://www.isi.edu/nsnam/ns/. Network Simulator 2, 1995.

[18] IEEE802.11b. Wireless LAN medium access control (MAC) and physical layer (PHY) specification: Higher-speed physical layer extension in the $2.4 \mathrm{GHz}$ band, 1999 .

[19] IEEE802.11e. 802.11e-2005 IEEE standard for information technology telecommunications and information exchange between systems local and metropolitan area networks specific requirements part 11: Wireless LAN medium access control (MAC) and physical layer (PHY) specifications:
Amendment 8: Medium access control (MAC) quality of service enhancements, 2005.

[20] I. Inanc, F. Keceli, and E. Ayanoglu. An adaptive multimedia QoS scheduler for IEEE 802.11e wireless LANs. In Proceedings of IEEE ICC, Istanbul, Turkey, June 2006.

[21] J. R. Jackson. Scheduling a production line to minimize maximum tardiness. Research Report 43, University of California, Los Angeles, CA, 1955. Management Science Research Project.

[22] C. Kuan and K. Dimyati. Utilization model for HCCA/EDCA mixed mode in IEEE 802.11e. ETRI Journal, 29(6):829-831, Dec. 2007.

[23] W. K. Lai, C. Shien, and C. Jiang. Adaptation of HCCA/EDCA ratio in IEEE 802.11 for improved system performance. Int. J. on Innovative Computing, Inf. and Contr., 5(11(B)):4177Ü-4188, Nov. 2009.

[24] P. Larcheri and R. L. Cigno. Scheduling in 802.11e: Open-loop or closed-loop? In Proceedings of IFIP WONS, Les Ménuires, France, Jan. 2006.

[25] C. L. Liu and J. W. Layland. Scheduling algorithms for multiprogramming in a hard-real-time environment. J. ACM, 20:46-61, Jan. 1973.

[26] S. Lu, V. Bharghavan, and R. Srikant. Fair scheduling in wireless packet networks. IEEE/ACM Trans. Net., 7(4):473-489, Aug. 1999.

[27] S. Mangold, S. Choi, P. May, O. Klein, G. Hiertz, and L. Stibor. IEEE 802.11e wireless LAN for quality of service. In Proceedings of European Wireless, volume 1, pages 32-39, Florence, Italy, Feb. 2002. invited paper.

[28] L. Palopoli, R. L. Cigno, and A. Colombo. Control and optimization of HCCA 802.11e access scheduling. In Proceedings of IEEE CDC, New Orleans, LA, Dec. 2007.

[29] A. L. Ruscelli, G. Cecchetti, A. Alifano, and G. Lipari. Enhancement of QoS support of HCCA schedulers using EDCA function in IEEE 802.11e networks. Ad Hoc Networks, 10(2):147-161, 2012. Special Issue on Recent advances in analysis and deployment of IEEE 802.11e and IEEE $802.11 \mathrm{p}$ protocol families.

[30] A. L. Ruscelli, G. Cecchetti, A. Mastropaolo, and G. Lipari. A greedy reclaiming scheduler for IEEE 802.11e HCCA networks. In Proceedings of ACM MSWIM, Miami, Florida, Nov. 2011.

[31] V. A. Siris and C. Courcoubetis. Resource control for the EDCA and HCCA mechanisms in IEEE 802.11e networks. In Proceedings of WiOpt, pages 22-27, Boston, Massachusetts, 2006.

[32] D. Skyrianoglou, N. Passas, and A. K. Salkintzis. ARROW: An efficient traffic scheduling algorithm for IEEE 802.11e HCCA. IEEE Transaction on Wireless Communications, 5(12):3558-3567, Dec. 2006.

[33] S.-L. Tsao. Extending Earliest Due Date scheduling algorithms for wireless networks with location-dependent errors. In Proceedings of 52nd IEEE VTC-Fall, volume 1, pages 223-228, Boston, MA, Sept. 2000. 